\title{
OBSERVATION OF SN1987A BY NEUTRINO LIGHT
}

\author{
F. REINES \\ Physics Department, University of California at Irvine, Irvine, CA 92717, USA
}

and

J. VANDERVELDE

University of Michigan, Ann Arbor, MI 48109, USA

Co-Spokesmen IMB Collaboration

On the 54th day of 1987 an event occurred on Earth which gave reality to the fifty-five year old dreams of Baade, Zwicky, and Landau - an event which confirmed thoughts of Chandrasekhar and Bethe. Also confirmed were the computer-based calculations of Colgate, Arnett, Burrows, Lattimer, Weaver, Woosley, Wilson and many others.

The event was the arrival of a neutrino pulse that had been rushing towards the Earth for $170000 \mathrm{yr}$. Had it arrived a few years earlier it would have gone unrecorded. Had its source in the Large Magellanic Cloud been much further away, it would have been too feeble to see. $\sim 10^{16}$ neutrinos passed through the IMB detector (in the United States) in a few seconds. Only eight made recorded interactions. Simultaneously the Kamiokande detector (in Japan) recorded eleven. These numbers are in remarkable accord with the expectations of the above thinkers. It is a masterful triumph of the human intellect.

Such pulses may pass our way every $10-100$ years. ${ }^{*)}$ The chance that any more will be recorded may not be high since it requires the maintenance of large, complex detectors over these very long time periods.

The detectors which recorded these events consisted of large masses of water viewed by many photomultiplier tubes located deep underground. $\left.{ }^{* *}\right)$

The events seen in the detectors are, individually, not very spectacular. In the IMB detector, for example, they have energies of only $20-40 \mathrm{MeV}$, close to that detector's threshold of visibility. An electron of $20 \mathrm{MeV}$ will only light up approximately two dozen of the detector's 2048 phototubes. Such a signal must be picked out from a background due to radioactivity, electronic noise, and neutrino interactions from the atmospheric cosmic ray background. By appropriate filtering one can reduce the data to the cosmic ray neutrino rate, which is about two events per week in the $20-50 \mathrm{MeV}$ energy band. A pulse of eight such events in six seconds is, therefore, truly extraordinary. This is what was recorded by IMB and similarly by a sequence of pulses in Kamiokande on February 23, many powers of ten above that expected from statistical fluctuations in the neutrino background.

*) Present experimental limits are $\$ 1$ per year.

${ }^{* *)}$ Details of these detectors may be found in publications of the two groups (Phys. Rev. Lett. April 6, 1987). 
A representation of one of the IMB events is shown in fig. 1. The view is from outside the detector looking northeast, in the general direction of the neutrinos from the supernova. The slashes represent photoelectrons counted in the phototubes hit by Cherenkov light from the event. The light comes from energetic charged particles made by neutrinos interacting with protons and electrons in the detector.

Characteristics of the eight events recorded by IMB are given in table 1. These data, along with the eleven events seen by the Kamiokande group, are being used to draw a variety of interesting inferences regarding both the Supernova and properties of the neutrino.

1. Supernova neutrino core "temperature".

2. Neutrino luminosity at the source.

3. Upper limits for the electron neutrino mass.

4. Neutrino stability.

As for the future of SN1987A, if there is a neutron star remaining at the site of the explosion, its rapid spin and high magnetic fields could give rise to an intense flux of particles with extremely high energies. Thus we are looking, at present, for neutrinos in the $\mathrm{GeV}$ and $\mathrm{TeV}$ energy range. These would be made manifest by the production of high-energy muons in the rock below the detector and the

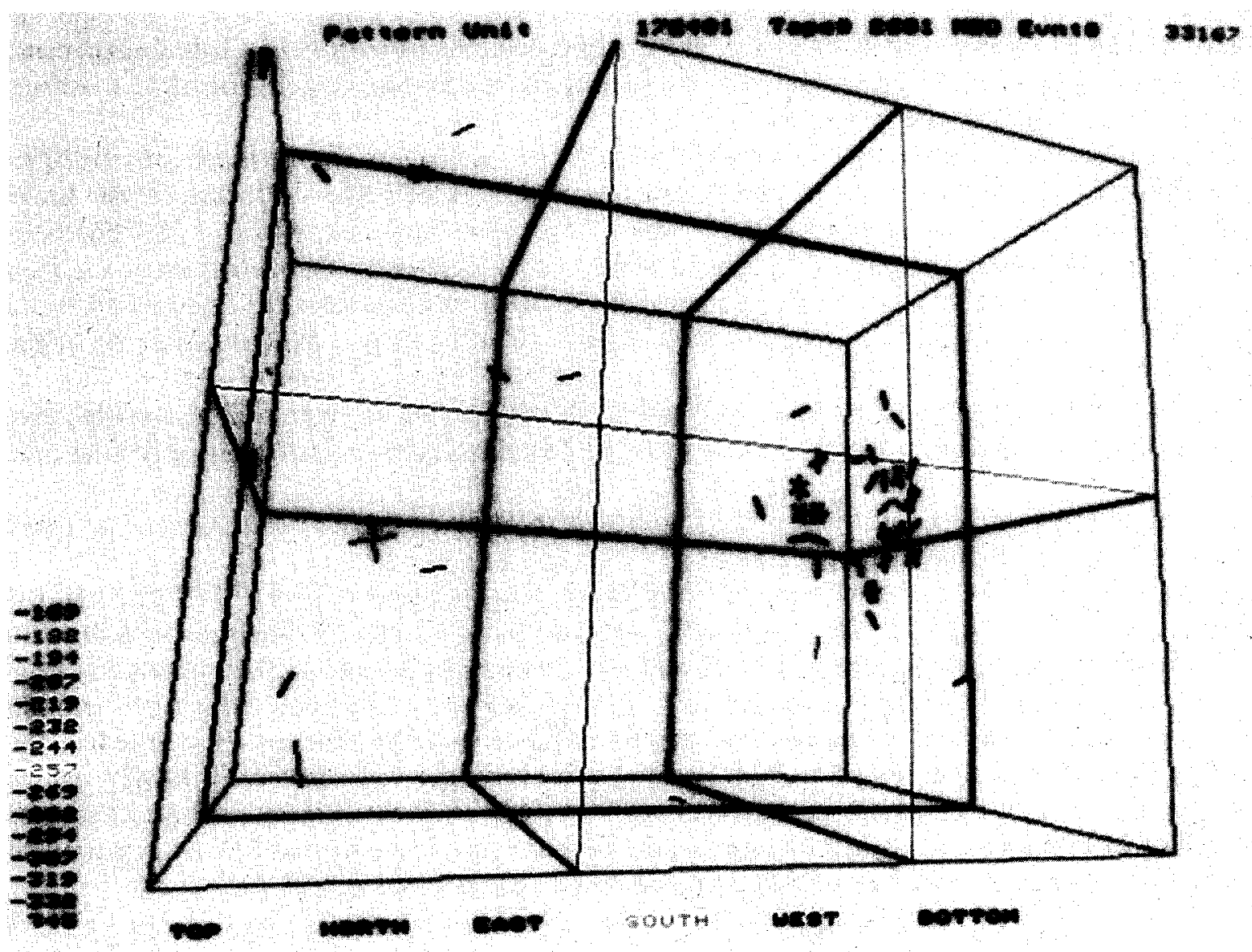

Fig. 1. Neutrino event from SN1987A in IMB detector. View is toward north-east corner. 
Table 1

SN1987A neutrino events in IMB detector

\begin{tabular}{|c|c|c|c|c|}
\hline Event & $\begin{array}{l}\text { Time (UT) } \\
23 \text { Feb. } 1987^{\text {a) }}\end{array}$ & $\begin{array}{l}\text { Measured energy } \\
(\mathrm{MeV})\end{array}$ & $\begin{array}{l}\text { Polar angle }{ }^{c)} \\
(\mathrm{deg})\end{array}$ & $\begin{array}{l}\left.\text { Antineutrino energy }{ }^{d}\right) \\
(\mathrm{MeV})\end{array}$ \\
\hline 1 & $7: 35: 41.374$ & $38 \pm 7$ & $80 \pm 10$ & $41 \pm 7$ \\
\hline 2 & $7: 35: 41.786$ & $37 \pm 7$ & $44 \pm 15$ & $39 \pm 7$ \\
\hline 3 & $7: 35: 42.024$ & $28 \pm 6$ & $56 \pm 20$ & $30 \pm 6$ \\
\hline 4 & $7: 35: 42.515$ & $39 \pm 7$ & $65 \pm 20$ & $42 \pm 7$ \\
\hline 5 & $7: 35: 42.936$ & $36 \pm 9$ & $33 \pm 15$ & $38 \pm 9$ \\
\hline 6 & $7: 35: 44.058$ & $36 \pm 6$ & $52 \pm 10$ & $38 \pm 6$ \\
\hline 7 & $7: 35: 46.384$ & $19 \pm 5$ & $42 \pm 20$ & $21 \pm 5$ \\
\hline 8 & $7: 35: 46.956$ & $22 \pm 5$ & $104 \pm 20$ & $24 \pm 5$ \\
\hline
\end{tabular}

a) Absolute UT is accurate to $\pm 50 \mathrm{~ms}$. Relative times are accurate to the nearest millisecond.

b) Additional systematic error in energy scale estimated to be $\pm 10 \%$.

${ }^{c)}$ Angle with respect to direction away from SN1987A. Angle errors include multiple scattering and event reconstruction.

${ }^{d)}$ Assumes events are due to $\bar{v}+\mathrm{p} \rightarrow \mathrm{e}^{+}+\mathrm{n}$ on free protons.

subsequent "upward muon" events pointing back to the Large Magellanic Cloud. Such an observation would rival the importance of the original neutrino pulse.

As exciting as it would be to see the supernova in this new way, it is even more exciting to realize that the essential features of the initial explosion are so well described by the supernova theory put forth by Hans A. Bethe and his theoretical colleagues. 